'Vicerrectoría de Investigación y Postgrado, Universidad Católica del Maule, Curicó, Chile. ¿Universidad Nacional de Mar del Plata, Argentina. aPhD Lingüística, 1 Investigadora de Postdoctorado

bInvestigador en CONICET. Candidato a Doctor en Administración Investigador en CONICET, Master in Business Administration.

Recibido el 29 de mayo de 2017, aceptado el 24 de octubre de 2017.

Este trabajo fue financiado en todas sus etapas por CONICYT, proyecto FONDECYT

\# 3160104/2016 titulado

Comunicación e Interculturalidad en Instituciones Públicas de la Salud en Chile.

Los autores no declaran conflictos de interés

Correspondencia a: Dra. Mariana Lazzaro-Salazar Vicerrectoría de Investigación y Postgrado, Universidad Católica del Maule Carmen 284, Curicó, Chile. mlazzaro@ucm.cl lazzaromari@gmail.com

\section{Riesgos psicosociales e integración profesional de médicos extranjeros: Un estudio sobre el manejo del conflicto en Chile}

\author{
MARIANA LAZZARO-SALAZAR ${ }^{1, \mathrm{a}}$, LUCAS J. PUJOL-COLS ${ }^{2, \mathrm{~b}}$
}

Background: The way in which communicative conflicts are confronted, as part of the acculturation process, can constitute a psychosocial risk factor that may affect the effectiveness of health care delivery. Aim: To describe the levels of dominance, adaptability and evasion as indicators of the way in which conflict situations are faced in the workplace by foreign physicians in the management of communicative conflicts. Materials and Methods: A self-report questionnaire was answered by 51 foreign physicians (63\% women, 76\% Venezuelans, 64\% aged between 31 and 40 years old) who work in public healthcare institutions in a southern region of Chile. Results: The highest scores were recorded for the adaptable style of conflict management (69\%), while the lowest scores were recorded for the evasive style (6\%). Higher levels of dominance were identified for women than for men. There was a positive association between dominance and time progression, where the level of dominance increased as participants gained greater professional experience and workplace seniority. Conclusions: The foreign physicians who participated in this study have a clear constructive attitude towards the negotiation of conflicts. However, this effort to adapt can also become a psychosocial risk factor that affects professional integration in intercultural workplace contexts.

(Rev Med Chile 2017; 145: 1300-1307)

Key words: Acculturation; Communication; Emigrants and Immigrants; Mental Health; Occupational Health.

L a comunicación entre médicos es un aspecto crucial para el funcionamiento exitoso de los equipos de trabajo en la medicina hospitalaria ${ }^{1}$. Como señalan Braš et $\mathrm{al}^{2}$, la comunicación efectiva juega un rol fundamental en la medicina moderna ya que es primordial para la colaboración profesional y el aseguramiento de la calidad del servicio hospitalario ${ }^{1}$. Entre las habilidades de comunicación inter-profesional se encuentran la presentación de casos clínicos ${ }^{3}$ y derivaciones, cuyas prácticas médicas a menudo varían de una institución a otra, y más aún al tratarse de un país a otro.

Esta última consideración es particularmente relevante para la realidad actual de las organizaciones de la salud alrededor del mundo, las que están experimentando profundos cambios demográficos en sus poblaciones profesionales como resultado de masivos procesos migratorios que terminan por conformar un sinfín de hospitales interculturales 5 . 
En este complejo contexto profesional, los conflictos derivados de la comunicación intercultural pueden, desde la perspectiva de la práctica médica, provocar disfunciones que incrementen el riesgo de negligencia médica ${ }^{3}$ mientras que, desde una perspectiva relacional, estos incrementan los factores de riesgo psicosocial, es decir aquellas demandas laborales que por exceso, defecto o combinación pueden convertirse en una amenaza para la integridad física, social y/o psicológica de los individuos ${ }^{6}$, a los que se ven expuestos médicos extranjeros y nacionales.

Con respecto a esto último, la incorporación a un equipo de trabajo en un país extranjero suele constituir una experiencia altamente estresante que le exige a los migrantes la movilización de una gran diversidad de recursos y estrategias con el propósito de adaptarse a las normas y valores imperantes de la cultura receptora ${ }^{7}$, fenómeno comúnmente denominado aculturación ${ }^{8}$. Este proceso de integración tiende a acrecentar los factores de riesgo psicosocial a los que tanto médicos migrantes como nacionales deben enfrentarse ${ }^{9}$. La incapacidad de los médicos (en términos de recursos personales) para hacer frente a estas nuevas demandas relacionales puede resultar en ansiedad y estrés laboral (o estrés por aculturación ${ }^{10,11}$ ) y, esto último, al desarrollo de relaciones insatisfactorias y a un ambiente laboral poco saludable ${ }^{12}$. A su vez, es muy posible que lo anterior afecte la satisfacción laboral de ambos grupos de médicos ${ }^{13}$ $y$, como resultado, su bienestar ${ }^{14}$ tanto desde el punto de vista de su salud física ${ }^{15}$ como mental ${ }^{16,17}$.

Las preocupaciones de este escenario globalizado no son ajenas a la realidad de las instituciones públicas de la salud de Chile. Según datos publicados por la Organización para la Cooperación Económica y Desarrollo ${ }^{18}$, Chile registra una de las tasas más bajas de médicos por habitante $(1,9$ médicos por cada 1.000 habitantes en 2013, comparado con 6,2 en Grecia) y de graduados en medicina $(7,8$ graduados por cada 100.000 habitantes, comparados con 19,6 en Austria). Naturalmente, esta situación tiende a traducirse en una escasez de médicos nacionales disponibles para la atención en el sistema público lo que atrae, como resultado, a una creciente población de médicos de diversos orígenes geográficos y culturales. No resulta sorprendente, entonces, que $4,7 \%$ de la población inmigrante de Chile se encuentre constituida por profesionales de la salud, muchos de los cuales son médicos ${ }^{19}$. Sin embargo, exceptuando las cifras de médicos extranjeros que aprueban/desaprueban el examen nacional para la práctica médica (Examen Único Nacional de Conocimientos de Medicina) ${ }^{20}$, el conocimiento sobre este reciente fenómeno cultural en las instituciones públicas de la salud en Chile es todavía escaso.

En este contexto, las altas implicancias de la comunicación entre médicos en un contexto cada vez más intercultural, como es el de las instituciones públicas de la salud de Chile, hacen de los estilos de manejo de conflictos un tema de estudio muy relevante. Así, este estudio se propone describir las actitudes hacia el conflicto comunicacional a través de la exploración de los estilos de manejo de conflicto de acuerdo a niveles de dominancia (control ejercido sobre la situación al imponer su estilo propio de manejo de conflicto), adaptabilidad (estilo de negociación para la resolución de conflictos) y evasión (estilo que favorece la no confrontación) como un indicador de la forma en la que se afrontan las situaciones conflictivas en el ámbito laboral en un sentido más amplio ${ }^{21,22}$. Además, se analiza la influencia de un conjunto de variables sociodemográficas sobre el nivel de dominancia, adaptabilidad y evasión de los médicos participantes, para luego discutir las posibles implicancias del nivel de adaptabilidad como posible factor de riesgo psicosocial en instituciones interculturales de la salud.

\section{Material y Método}

\section{Diseño}

Se utilizó un diseño transversal y se realizó una encuesta de auto-percepción de conflictos comunicacionales a médicos extranjeros que trabajan en instituciones públicas de la salud de la VII Región de El Maule, Chile. Aunque los datos aquí presentados forman parte de un estudio más amplio sobre la comunicación intercultural en instituciones públicas de la salud que también comprende las regiones de Antofagasta y Magallanes, este artículo presenta los resultados de la primera etapa (de tres) del estudio en la región del Maule.

\section{Participantes}

Un total de 51 médicos extranjeros que trabajan en instituciones públicas de la salud de la VII Región de El Maule participaron en este estudio (Tabla 1). 
Tabla 1. Estructura de la muestra

\begin{tabular}{|c|c|c|}
\hline Variable & Categoría & $\begin{array}{l}\text { Frecuencia } \\
\text { porcentual }\end{array}$ \\
\hline \multirow[t]{2}{*}{ Género } & Masculino & $37,25 \%$ \\
\hline & Femenino & $62,75 \%$ \\
\hline \multirow[t]{3}{*}{ Edad } & Menos de 30 años & $7,89 \%$ \\
\hline & Entre 31 y 40 años & $64,70 \%$ \\
\hline & Más de 40 años & $27,46 \%$ \\
\hline \multirow[t]{5}{*}{ País de origen } & Venezuela & $76,47 \%$ \\
\hline & Colombia & $11,76 \%$ \\
\hline & Ecuador & $3,92 \%$ \\
\hline & Cuba & $3,92 \%$ \\
\hline & Otros & $3,92 \%$ \\
\hline \multirow{9}{*}{$\begin{array}{l}\text { Especialidad } \\
\text { médica }\end{array}$} & Anestesiólogos & $23,53 \%$ \\
\hline & Médicos generales & $21,57 \%$ \\
\hline & Pediatras & $17,75 \%$ \\
\hline & Intensivistas & $9,80 \%$ \\
\hline & Cirujanos generales & $7,84 \%$ \\
\hline & Ginecólogos & $3,92 \%$ \\
\hline & Médicos de familia & $3,92 \%$ \\
\hline & Radiólogos & $3,92 \%$ \\
\hline & Otros & $7,88 \%$ \\
\hline \multirow{2}{*}{$\begin{array}{l}\text { Dedicación } \\
\text { horaria semanal }\end{array}$} & Tiempo completo & $76,47 \%$ \\
\hline & Tiempo parcial & $23,59 \%$ \\
\hline \multirow{3}{*}{$\begin{array}{l}\text { Años de ejercicio } \\
\text { profesional }\end{array}$} & $1-10$ años & $41,18 \%$ \\
\hline & 11-20 años & $49,02 \%$ \\
\hline & 21 años o más & $9,80 \%$ \\
\hline
\end{tabular}

\section{Instrumento}

La encuesta sobre conflicto comunicacional fue diseñada adaptando la Prueba Holandesa para el Manejo de Conflictos (The Dutch Test for Conflict Handling, DUTCH) validada por De Dreu et $\mathrm{al}^{22,23}$. La encuesta consta de 47 ítems agrupados en 3 dimensiones centrales: 1) conflicto comunicativo; 2) estilos de manejo de conflicto comunicativo que incluyen: (a) dominante, (b) adaptable y (c) evasivo; y 3) el tipo de lenguaje utilizado. Estas tres dimensiones se presentan en dos escalas Likert de 5 puntos que constituyen las dos secciones de la encuesta, una que mide el nivel percibido de conflicto comunicativo y otra que mide la frecuencia con la que los médicos extranjeros perciben los conflictos comunicativos. El presente estudio reporta los resultados de la segunda dimensión de la encuesta.

\section{Procedimientos}

Siguiendo las normas éticas establecidas en la Declaración de Helsinki, se obtuvo la aprobación para la realización del estudio del Comité de Ética de la Universidad Católica del Maule (Acta \# 28/2015). Los participantes fueron contactados por correo electrónico y/o por mensaje de texto y se proporcionó un enlace para llenar la encuesta en línea. La encuesta se encuentra precedida por una descripción del estudio y un formulario de consentimiento informado. Solo se otorgó acceso a la encuesta en línea cuando el consentimiento informado para participar en este estudio fue aceptado. Las respuestas a la encuesta fueron anónimas y cualquier información personal que permita identificar a los participantes y las instituciones donde trabajan es estrictamente confidencial. Los datos reportados aquí fueron recolectados entre noviembre de 2016 y abril de 2017.

\section{Análisis}

Los niveles de dominancia, adaptabilidad y evasión frente al conflicto comunicacional fueron calculados como la media aritmética de los ítems que componen cada una de las escalas. La fiabilidad fue evaluada en términos de su consistencia interna, para lo cual se calculó el Coeficiencia Alfa de Cronbach. Adicionalmente, se analizó la sensibilidad de dicho estimador ante modelos alternativos de cantidad y composición de ítems. Para cada escala, se calcularon medidas de tendencia central (media) y de dispersión (desvío estándar), además de las correlaciones observadas entre las mismas. Finalmente, se analizó la influencia de distintas variables de índole sociodemográfica sobre los niveles promedio de dominancia, adaptabilidad y evasión observados en los participantes. Para ello se emplearon las pruebas estadísticas de diferencia de medias t de Student y ANOVA de una vía.

\section{Resultados}

\section{Variables sociodemográficas}

En la Tabla 1, se observan los resultados de los datos sociodemográficos de los participantes. Con relación a las razones que han motivado el traslado de estos médicos a Chile se destacan: la seguridad personal $(76,47 \%)$, razones políticas del país de origen (52,94\%), las económicas $(49,02 \%)$, y el desarrollo profesional (19,61\%). El tiempo promedio que los participantes han permanecido 
en el país es de 38 meses y la antigüedad promedio registrada en el empleo actual de 31 meses. Se observa que, en promedio, los participantes permanecen 4 meses en el país hasta encontrar su primer empleo y que sólo 29,41\% de los mismos ingresó al país con un contrato laboral previo. Se destaca que $37,25 \%$ de los participantes reporta haber experimentado una reducción en su status ocupacional, al desempeñarse actualmente en un cargo con menor jerarquía que la poseída en su país de origen.

\section{Actitudes hacia el conflicto comunicacional}

La confiabilidad de las tres escalas (dominancia, adaptabilidad y evasión) resultó con un Coeficiente Alfa de Cronbach aceptable (Tabla 2), registrando correlaciones no significativas entre sí, lo que refleja tres actitudes diferentes frente al conflicto comunicacional.

En la Tabla 3 se observa que el puntaje más alto se registra para el estilo adaptable, mientras que el más bajo se registra para el estilo evasivo.

\section{Influencia de variables sociodemográficas en la actitud hacia el conflicto comunicacional}

En esta sección del estudio se analizó la influencia de las variables: edad, género, antigüedad en la profesión, dedicación horaria semanal, estudios en el extranjero, reducción en el status ocupacional, tiempo de estadía en el país, tiempo transcurrido en el país luego obtener el primer empleo y la antigüedad en el empleo sobre los niveles de dominancia, adaptabilidad y evasión. En la Tabla 4 se describen los niveles promedio y desviaciones estándar para cada uno de los subgrupos de las variables sociodemográficas analizadas en relación con los niveles de dominancia, adaptabilidad y evasión.

En términos de la influencia de las variables (Tabla 5), se observa que solo las variables género del participante, la antigüedad en el ejercicio de la profesión, la cantidad de meses transcurridos desde el primer empleo en Chile y la antigüedad en el empleo actual mostraron una influencia significativa sobre el nivel de dominancia. Para la variable género, las mujeres registran niveles de dominancia promedio mayores que los hombres. En el caso de la antigüedad en el ejercicio de la profesión, se observa que aquellos médicos con una antigüedad de entre 11 y 20 años reportan ser más dominantes en términos comunicacionales que aquellos con una antigüedad igual o menor a 10 años. Además, la cantidad de meses transcurridos desde la obtención del primer puesto de trabajo en Chile muestra que los médicos extranjeros con una antigüedad reportada menor a 12 meses poseen un nivel de dominancia significativamente menor que aquellos que llevan entre 12 y 23 meses en el país. Finalmente, el grupo con una antigüedad en el empleo actual de entre 12 y 23 meses muestra mayores niveles de dominancia que el grupo con una antigüedad menor a 12 meses.

Tabla 2. Descriptivos y correlaciones entre escalas de orientación hacia el conflicto comunicacional

\begin{tabular}{|lccccccc|}
\hline Orientación & M & DE & Mínimo & Máximo & $\mathbf{1}$ & $\mathbf{2}$ & $\mathbf{3}$ \\
\hline 1. Dominancia & 2,50 & 0,71 & 0,6 & 3,6 & $\mathbf{0 , 6 6}$ & & \\
\hline 2. Adaptabilidad & 3,12 & 0,63 & 1,7 & 4,5 & $-0,21$ & $\mathbf{0 , 7 7}$ & \\
\hline 3. Evasión & 1,88 & 0,70 & 0,14 & 3 & $-0,09$ & 0,03 & $\mathbf{0 , 6 7}$ \\
\hline
\end{tabular}

Nota. $M=$ Media, DE = Desvío estándar. La fiabilidad de las escalas (expresadas en términos de su Coeficiente Alfa de Cronbach) se encuentra indicada sobre la diagonal principal en negritas. Las correlaciones entre las escalas 1, 2 y 3 son, en todos los casos, no significativas.

Tabla 3. Distribución de frecuencias de acuerdo a la orientación hacia el conflicto comunicacional

\begin{tabular}{|lcccc|}
\hline Orientación & Bajo & Moderado & Alto & Orientación dominante \\
\hline Dominancia & $11,76 \%$ & $72,55 \%$ & $15,69 \%$ & $25,49 \%$ \\
\hline Adaptabilidad & $0,00 \%$ & $66,67 \%$ & $33,33 \%$ & $68,63 \%$ \\
\hline Evasión & $39,22 \%$ & $60,78 \%$ & $0,00 \%$ & $5,88 \%$ \\
\hline
\end{tabular}

Nota. $\mathrm{N}=51$. Categorías: Baja = de 0 a 1,66; Moderada = de 1,67 a 3,33; Alta = de 3,34 a 5. 
Tabla 4. Medias y desvíos estándar por grupo

\begin{tabular}{|c|c|c|c|c|c|c|}
\hline \multirow[t]{2}{*}{ Subgrupos } & \multicolumn{2}{|c|}{ Dominancia } & \multicolumn{2}{|c|}{ Adaptabilidad } & \multicolumn{2}{|c|}{ Evasión } \\
\hline & M & DE & M & DE & $\mathbf{M}$ & DE \\
\hline \multicolumn{7}{|l|}{ Edad } \\
\hline Menor de 35 años & 2,50 & 0,79 & 3,21 & 0,73 & 1,69 & 0,77 \\
\hline Mayor o igual de 36 años & 2,49 & 0,67 & 3,07 & 0,58 & 1,98 & 0,66 \\
\hline \multicolumn{7}{|l|}{ Género } \\
\hline Masculino & 2,26 & 0,75 & 3,32 & 0,53 & 1,77 & 0,69 \\
\hline Femenino & 2,63 & 0,66 & 3,00 & 0,67 & 1,94 & 0,71 \\
\hline \multicolumn{7}{|c|}{ Años de ejercicio de la profesión } \\
\hline $1-10$ & $2,26^{a}$ & 0,77 & $3,30^{a}$ & 0,61 & 1,79 & 0,67 \\
\hline $11-20$ & $2,74^{a}$ & 0,56 & $3,06^{a}$ & 0,61 & 1,94 & 0,77 \\
\hline+21 & 2,24 & 0,82 & 2,66 & 0,65 & 1,94 & 0,59 \\
\hline \multicolumn{7}{|c|}{ Dedicación horaria semanal } \\
\hline Menos de $40 \mathrm{~h}$ & 2,73 & 0,79 & 3,27 & 0,65 & 1,85 & 0,53 \\
\hline Más de $40 \mathrm{~h}$ & 2,42 & 0,68 & 3,07 & 0,63 & 1,89 & 0,76 \\
\hline \multicolumn{7}{|l|}{ Estudios en el extranjero } \\
\hline Sí & 2,42 & 0,79 & 3,09 & 0,65 & 1,79 & 0,73 \\
\hline No & 2,55 & 0,66 & 3,14 & 0,63 & 1,94 & 0,69 \\
\hline \multicolumn{7}{|l|}{ Pérdida de status } \\
\hline Sí & 2,61 & 0,61 & 2,98 & 0,54 & 1,85 & 0,72 \\
\hline No & 2,40 & 0,76 & 3,20 & 0,68 & 1,89 & 0,71 \\
\hline \multicolumn{7}{|c|}{ Tiempo de estadía en el país } \\
\hline Menos de 12 meses & 2,18 & 0,94 & 3,04 & 0,58 & 2,00 & 0,65 \\
\hline Entre 12 y 23 meses & 2,83 & 0,75 & 3,09 & 0,68 & 2,21 & 0,74 \\
\hline Entre 24 y 35 meses & 2,47 & 0,67 & 3,33 & 0,82 & 1,55 & 0,70 \\
\hline 36 meses o más & 2,52 & 0,60 & 3,04 & 0,53 & 1,88 & 0,69 \\
\hline \multicolumn{7}{|c|}{ Tiempo transcurrido desde el primer empleo } \\
\hline Menos de 12 meses & $2,05^{b}$ & 0,85 & 3,26 & 0,68 & 1,94 & 0,58 \\
\hline Entre 12 y 23 meses & $2,87^{b}$ & 0,59 & 2,77 & 0,71 & 2,05 & 0,79 \\
\hline Entre 24 y 35 meses & 2,50 & 0,73 & 3,26 & 0,63 & 1,45 & 0,80 \\
\hline 36 meses o más & 2,61 & 0,52 & 3,11 & 0,54 & 2,03 & 0,60 \\
\hline \multicolumn{7}{|c|}{ Antigüedad en el empleo actual } \\
\hline Menos de 12 meses & $2,10^{c}$ & 0,85 & 3,18 & 0,66 & 1,95 & 0,55 \\
\hline Entre 12 y 23 meses & $3,00^{c}$ & 0,43 & 3,01 & 0,79 & 1,87 & 0,81 \\
\hline 24 meses o más & 2,51 & 0,60 & 3,13 & 0,57 & 1,84 & 0,76 \\
\hline
\end{tabular}

Nota. $\mathrm{N}=51 . \mathrm{M}=$ Media, $\mathrm{DE}=$ Desviación estándar. ${ }^{\mathrm{a}}=$ Prueba post hoc HSD de Tukey, $\mathrm{p}<0,10 .{ }^{\mathrm{b}}=$ Prueba post hoc HSD de Tukey, $p<0,05 .{ }^{c}=$ Prueba post hoc HSD de Tukey, $p<0,01$.

En relación con el nivel de adaptabilidad, el análisis muestra que el género, la antigüedad en la profesión y la antigüedad como empleado en Chile son factores que influyen sobre la adaptabilidad frente al conflicto comunicacional. En cuanto al género, se observa que los hombres reportan ser más adaptables que las mujeres. También se observa que la adaptabilidad disminuye a medida que aumentan los años de ejercicio profesional, siendo el mayor nivel el del grupo de participantes con experiencia menor a 10 años y el menor para el grupo de aquellos con más de 20 años. Con relación a la antigüedad como empleado en Chile, se observa que el grupo con una antigüedad de entre 12 y 23 meses registra el menor nivel de adaptabilidad frente al conflicto comunicacional. Finalmente, en este estudio no se identificó la influencia de ninguna variable sociodemográfica sobre el nivel de evasión. 
Tabla 5. Incidencia sociodemográfica en la orientación frente al conflicto comunicacional

\begin{tabular}{|lcccccc|}
\hline Orientación & \multicolumn{2}{c}{ Dominancia } & \multicolumn{2}{c}{ Adaptabilidad } & \multicolumn{2}{c|}{ Evasión } \\
Variables & $\mathbf{t} / \mathbf{F}$ & $\mathbf{p}$ & $\mathbf{t / F}$ & $\mathbf{p}$ & t/F & $\mathbf{p}$ \\
\hline Edad & 0,03 & 0,98 & 0,73 & 0,47 & $-1,41$ & 0,17 \\
Género & 1,85 & $0,07^{*}$ & $-1,81$ & $0,08^{*}$ & 0,80 & 0,43 \\
\hline Años de ejercicio de la profesión & 3,22 & $0,05^{* *}$ & 2,44 & $0,10^{*}$ & 0,27 & 0,77 \\
Horas semanales & 1,34 & 0,19 & 0,93 & 0,18 & $-0,18$ & 0,86 \\
\hline Estudios en el extranjero & 0,65 & 0,52 & 0,29 & 0,77 & 0,74 & 0,46 \\
Pérdida de status ocupacional & $-1,22$ & 0,23 & 1,21 & 0,88 & 0,21 & 0,83 \\
\hline Tiempo de estadía en el país & 1,21 & 0,32 & 0,61 & 0,61 & 1,64 & 0,19 \\
\hline Antigüedad como empleado en Chile & 2,83 & $0,05^{* *}$ & 1,25 & 0,37 & 2,08 & 0,12 \\
\hline Antigüedad en el empleo actual & 5,59 & $0,01^{* * *}$ & 0,21 & 0,81 & 0,10 & 0,90 \\
\hline
\end{tabular}

Nota. $\mathrm{t} / \mathrm{F}=$ Valor del estadístico t o $\mathrm{F}$ según la prueba que corresponda, $\mathrm{p}=\mathrm{p}$-value. ${ }^{*} \mathrm{p}<0,10 ;{ }^{* *} \mathrm{p}<0,05 ;{ }^{* *} \mathrm{p}<0,01$.

\section{Discusión y conclusiones}

Este estudio contribuye a la investigación de, por una parte, la forma en la que los médicos extranjeros reportan afrontar las situaciones conflictivas en las instituciones públicas de salud en Chile, y la relación entre estas actitudes y ciertas variables sociodemográficas, y, por el otro lado, la consideración del posible impacto del nivel de adaptabilidad como factor de riesgo psicosocial en los procesos de aculturación e integración profesional.

Con respecto a la actitud hacia el manejo de conflicto de los médicos extranjeros que participaron de este estudio, se puede decir que el bajo porcentaje reportado para los estilos de evasión y dominancia muestra una actitud positiva hacia la resolución del conflicto, lo que a su vez se refleja en el alto porcentaje obtenido para el estilo de adaptabilidad. Esto último muestra que estos médicos tienen claramente un estilo de manejo de conflicto que favorece la negociación de estos, evidenciando una actitud constructiva que propicia la integración profesional en contextos laborales interculturales ${ }^{24,25}$. Esta actitud puede contribuir no solo a mantener relaciones laborales armoniosas ${ }^{26}$, sino que también a facilitar la transferencia de conocimiento médico, mejorar la calidad del servicio de salud y a disminuir la tasa de errores ${ }^{27-29}$.

Sin embargo, no se debe perder de vista que este esfuerzo por adaptarse también puede cons- tituir un factor de riesgo psicosocial que afecte de manera negativa a los médicos que reportan este estilo de manejo de conflictos. Como explica $\mathrm{Kim}^{30}$, en el proceso de aculturación son casi exclusivamente los inmigrantes quienes soportan la carga de hacer los ajustes. De este modo, las demandas psicológicas involucradas se convierten en un factor de riesgo psicosocial que requiere no sólo de esfuerzos cognitivos adicionales, sino también del manejo de emociones negativas que se experimenten como resultado de este proceso ${ }^{31}$. Estas exigencias pueden provocar sentimientos de angustia y estrés que, sostenido en el tiempo, pueden tener un efecto perjudicial sobre la salud física y mental de los que las experimenten ${ }^{10,32}$.

En este sentido, los resultados sobre la relación entre las actitudes hacia el conflicto y la variable de género muestran mayores niveles de dominancia en mujeres que en hombres por lo que no parece apresurado concluir que los hombres pueden ser más vulnerables al estrés por aculturación que las mujeres. Adicionalmente, los resultados muestran una asociación positiva entre la dominancia y el transcurso del tiempo, incrementándose el nivel de dominancia a mayor antigüedad profesional y laboral. Esto sugiere que a mayor tiempo transcurrido en el ejercicio de la profesión en el país y del cargo laboral, mayores posibilidades de que el proceso de adaptación y, por consecuente, de aculturación se encuentren más desarrollados por lo que los migrantes experimentan una mayor dominancia sobre las situaciones conflictivas. 
Como resultado, esto podría además a contribuir a disminuir los niveles de riesgos psicosociales.

Naturalmente, no es posible afirmar que la sola presencia de riesgos psicosociales produzca siempre resultados negativos para la salud y, en consecuencia, un impacto negativo en el entorno de trabajo intercultural, ya que los individuos pueden percibir el estrés de diferentes formas y en diferentes grados ${ }^{33}$. Sin embargo, el reflexionar sobre el posible impacto de los procesos de adaptación a los que se enfrentan los médicos inmigrantes sobre las relaciones interpersonales y los equipos médicos interculturales plantea la necesidad de realizar más investigaciones para abordar el estudio de las relaciones propuestas y el estrés que experimentan los médicos extranjeros en contextos laborales. Esto, además, abre la necesidad de estudiar el impacto de los procesos de aculturación de los médicos extranjeros sobre los médicos nacionales que de forma directa o indirecta acompañan estos procesos.

Por el momento, es importante enfatizar que es necesario desarrollar sistemas de salud culturalmente competentes ${ }^{34}$ para hacer frente a estos factores de riesgo psicosociales. Esto es aún más relevante en las primeras etapas del proceso de aculturación en el que los inmigrantes se enfrentan al doble reto de adaptarse tanto a las normas y valores culturales de un nuevo país como a la idiosincrasia del nuevo entorno laboral. En este sentido, la provisión de recursos organizacionales resulta esencial para la adaptación exitosa de estos inmigrantes y su inserción profesional.

\section{Referencias}

1. Schnoor J, Braehler E, Ghanem M, Heyde C. The impact of economic rationalization, prioritization and rationing on job satisfaction, motivation and team cohesion in hospitals: a survey among retired physician executives in Germany. Patient Safety in Surgery 2017; 11 (1): 1-10.

2. Braš M, Đor evi V, Ruiz-Moral R, Deveugele M, Ramalho R, Pype P. The Role of Communication. En: Mezzich J, Botbol M, Christodoulou G, Cloninger C, Salloum I. (eds) Person Centered Psychiatry. New York: Springer International Publishing 2016.

3. Porter R. Communication and counselling. En: Luesley D, Kilby M. (eds.) Obstetrics \& Gynaecology: An Evidence-based Text for MRCOG ( $3^{\text {rd }}$ ed.). London: CRC Press 2016.
4. McConnell D, Butow P, Tattersall M. Improving the letters we write: An exploration of doctor-doctor communication in cancer care. British Journal of Cancer 2015; 80 (3-4): 427.

5. Forbes Insights. Diversity and Inclusion: Unlocking Global Potential. Global Diversity Rankings by Country, Sector and Occupation. New York: Forbes 2012.

6. Melia J, Nogareda C, Lahera M, Duro A, Peiro J, Salanova M, Gracia D, de Bona J M, Bajo J, Martínez-Losa F. (Eds.) Perspectivas de Intervención en Riesgos Psicosociales. Evaluación de riesgos. Barcelona: Foment del Treball Nacional.

7. de Almeida Vieira Monteiro APT, Serra AVJ. Vulnerability to stress in migratory contexts: a study with eastern European immigrants residing in Portugal. Journal of Immigrant and Minority Health 2011;13 (4): 690-6.

8. Amer MM, Hovey JD. Socio-demographic differences in acculturation and mental health for a sample of 2 nd generation/early immigrant Arab Americans. Journal of Immigrant and Minority Health 2007; 9 (4): 335.

9. Bruhn JG, Chesney AP, Salcido R. Health and organizational issues in managing a multicultural work force. Family and Community Health 1995; 18 (2): 1-8.

10. Hovey JD, Magaña C. Acculturative stress, anxiety, and depression among Mexican immigrant farmworkers in the Midwest United States. Journal of Immigrant Health 2000; 2 (3): 119-31.

11. Urzúa MA, Heredia BO, Caqueo-Urizar A. (2016). Mental health and stress by acculturation in immigrants from South America in northern Chile. Rev Med Chile 2016; 144 (5): 563-70.

12. Kreitzer MJ, Wright D, Hamlin C, Towey S, Marko M, Disch J. Creating a healthy work environment in the midst of organizational change and transition. Journal of Nursing Administration 1997; 27 (6): 35-41.

13. Dierdorff EC, Morgeson FP. Getting what the occupation gives: Exploring multilevel links between work design and occupational values. Personnel Psychology 2013; 66 (3): 687-721.

14. Warr P. Work, happiness, and unhappiness. Mahwah: Lawrence Erlbaum; 2007.

15. Faragher EB, Cass M, Cooper CL. The relationship between job satisfaction and health: a meta-analysis. Occupational and Environmental Medicine 2005; 62 (2): 105-12.

16. Kammeyer-Mueller JD, Judge TA, Scott BA. The role of core self-evaluations in the coping process. Journal of Applied Psychology 2009; 94 (1): 177-95.

17. Tsaousis I, Nikolaou I, Serdaris N, Judge, TA. Do the core self-evaluations moderate the relationship between subjective well-being and physical and psychological 
health? Personality and Individual Differences 2007; 42 (8): 1441-52.

18. Acceso online: http://www.oecd.org/els/health-systems/ oecd-health-statistics-2014-frequently-requested-data. htm

19. Acceso online: http://observatorio.ministeriodesarrol.. ñlosocial.gob.cl/documentos/CASEN_2013_Inmigrantes_01_marzo.pdf

20. Acceso online: El Mercurio, 13/02/17 http://www. emol.com/noticias/Nacional/2017/02/13/844660/ Mas-de-mil-medicos-extranjeros-con-Eunacom-aprobado-no-trabajan-en-el-sector-publico.html

21. Ting-Toomey S, Gao G, Trubisky P, Yang Z, Soo Kim, $\mathrm{H}$, Lin SL, Nishida T. Culture, face maintenance, and styles of handling interpersonal conflict: A study in five cultures. International Journal of Conflict Management 1991; 2 (4): 275-96.

22. De Dreu CK, Evers A, Beersma B, Kluwer ES, Nauta A. A theory based measure of conflict management strategies in the workplace. Journal of Organizational Behavior 2001; 22 (6): 645-68.

23. Rahim MA. Managing conflict in organizations (3rd ed.). Westport: Quorum Books; 2001.

24. Saltman DC, O'dea NA, Kidd MR. Conflict management: a primer for doctors in training. Postgraduate Medical Journal 2006; 82 (963): 9-12.

25. Oetzel J, Dhar S, Kirschbaum K. Intercultural conflict from a multilevel perspective: Trends, possibilities, and future directions. Journal of Intercultural Communication Research 2007; 36 (3): 183-204.

26. Euwema MC, Van Emmerik IH. Intercultural compe- tencies and conglomerated conflict behaviors in intercultural conflicts. International Journal of Intercultural Relations 2007; 31 (4): 427-41.

27. Hewett DG, Watson BM, Gallois C. Communication between hospital doctors: Underaccommodation and interpretability. Language \& Communication 2015; 41: 71-83.

28. Cocksedge S, Barr N, Deakin C. Sharing information: Mixed-methods investigation of brief experiential interprofessional training for healthcare staff. Communication \& Medicine 2015; 12 (1): 1-12.

29. Hamilton H, Chou WYS. The Routledge handbook of language and health communication. London: Routledge; 2014.

30. Kim YY. Becoming intercultural: An integrative theory of communication and cross-cultural adaptation. London: Sage; 2001.

31. Moncada S, Llorens C, Kristensen T. Método ISTAS21 (CoPsoQ). Manual para la evaluación de riesgos psicosociales en el trabajo. Madrid: ISTAS; 2004.

32. Shen BJ, Takeuchi DT. A structural model of acculturation and mental health status among Chinese Americans. American Journal of Community Psychology 2001; 29 (3): 387-418.

33. Smith-Castro V. Acculturation and psychological adaptation. Westport: Greenwood Press; 2003.

34. Anderson LM, Scrimshaw SC, Fullilove MT, Fielding JE, Normand J. Task Force on Community Preventive Services. Culturally competent healthcare systems: a systematic review. American Journal of Preventive Medicine 2003; 24 (3): 68-79. 